\title{
Long-Term Follow-Up of Cognition and Mental Health in Adult Phenylketonuria: A PKU-COBESO Study
}

\author{
Rianne Jahja ${ }^{1}$ - Francjan J. van Spronsen ${ }^{1}$ Leo M. J. de Sonneville ${ }^{2} \cdot J^{2 a a p ~ J . ~ v a n ~ d e r ~ M e e r e ~}{ }^{3}$ Annet M. Bosch ${ }^{4}$. \\ Carla E. M. Hollak ${ }^{4} \cdot$ M. Estela Rubio-Gozalbo ${ }^{5}$ - Martijn C. G. J. Brouwers ${ }^{6}$ - Floris C. Hofstede ${ }^{7}$. \\ Maaike C. de Vries ${ }^{8} \cdot$ Mirian C. H. Janssen ${ }^{8}$ Ans T. van der Ploeg • Janneke G. Langendonk9 • \\ Stephan C. J. Huijbregts ${ }^{2,10}$
}

Received: 29 December 2016 / Accepted: 7 July 2017

(C) The Author(s) 2017. This article is an open access publication

\begin{abstract}
Cognitive and mental health problems in individuals with the inherited metabolic disorder phenylketonuria (PKU) have often been associated with metabolic control and its history. For the present study executive functioning (EF) was assessed in $21 \mathrm{PKU}$ patients during childhood ( $\mathrm{T} 1$, mean age 10.4 years, $\mathrm{SD}=2.0)$ and again in adulthood ( $\mathrm{T} 2$, mean age 25.8 years, $\mathrm{SD}=2.3$ ). At $\mathrm{T} 2$ additional assessments of $\mathrm{EF}$ in daily life and mental health were performed. Childhood (i.e. 0-12 years) blood phenylalanine was significantly related to cognitive flexibility, executive motor control, EF in daily life and mental health in adulthood (i.e. at T2). Patients with a greater increase in phenylalanine levels after the age of 12 performed more poorly on EF-tasks at T2. Group-based analyses showed that patients with phenylalanine $<360 \mu \mathrm{mol} / \mathrm{L}$ in childhood and phenylalanine $\geq 360 \mu \mathrm{mol} / \mathrm{L}$ from age 13 onwards $(n=11)$ had better cognitive flexibility and executive motor
\end{abstract}

Edited by Chandra Reynolds.

Francjan J. van Spronsen

f.j.van.spronsen@umcg.nl

Stephan C. J. Huijbregts

shuijbregts@fsw.leidenuniv.nl

1 University of Groningen, University Medical Center Groningen, Beatrix Children's Hospital, Groningen, The Netherlands

2 Department of Clinical Child and Adolescent Studies \& Leiden Institute for Brain and Cognition, Leiden University, Leiden, The Netherlands

3 University of Groningen, Department of Developmental and Clinical Neuropsychology, Groningen, The Netherlands

4 Academic Medical Center, Amsterdam, The Netherlands

5 University Hospital Maastricht and Laboratory Genetic Metabolic Diseases, Maastricht, The Netherlands control than those who had phenylalanine $\geq 360 \mu \mathrm{mol} / \mathrm{L}$ throughout life $(n=7)$, supporting the notion that phenylalanine should be below the recommended upper treatment target of $360 \mu \mathrm{mol} / \mathrm{L}$ during childhood for better outcome in adulthood. Despite some results indicating additional influence of phenylalanine levels between 13 and 17 years of age, evidence for a continued influence of phenylalanine levels after childhood on adult outcomes was largely lacking. This may be explained by the fact that the patients in the present study had relatively low phenylalanine levels during childhood (mean: $330 \mu \mathrm{mol} / \mathrm{L}$, range: 219 $581 \mu \mathrm{mol} / \mathrm{L}$ ) and thereafter (mean Index of Dietary Control at T2: $464 \mu \mathrm{mol} / \mathrm{L}$, range: $276-743 \mu \mathrm{mol} / \mathrm{L}$ ), which may have buffered against transitory periods of poor metabolic control during adolescence and early adulthood.

6 Division of Endocrinology and Metabolic Diseases, Department of Internal Medicine, University Hospital Maastricht, Maastricht, The Netherlands

7 Wilhelmina Children's Hospital, University Medical Center Utrecht, Utrecht, The Netherlands

8 University Medical Center St Radboud Nijmegen, Nijmegen, The Netherlands

9 Center for Lysosomal and Metabolic Diseases, Erasmus Medical Center, Rotterdam, The Netherlands

10 Department of Clinical Child and Adolescent Studies, Leiden University, Wassenaarseweg 52, P.O. Box 9555, 2300 RB Leiden, The Netherlands 
Keywords Phenylketonuria - Executive functioning . Executive motor control · Mental health · Adults . Longitudinal

\section{Introduction}

Notwithstanding the prevention of intellectual disability by decreasing blood phenylalanine (Phe) concentrations, the neurocognitive and psychosocial outcomes of patients with phenylketonuria (PKU; OMIM 212600) are on average still below the level of their healthy counterparts. PKU is a rare inborn error of metabolism, with a mean prevalence of $1: 10,000$, that is characterized by deficient hepatic enzyme phenylalanine hydroxylase (PAH) activity. PAH normally helps to convert Phe into tyrosine (Tyr), which is the precursor to L-dopa and consequently dopamine (Blau et al. 2010; van Spronsen et al. 2017). Untreated PKU patients show high blood Phe levels and low to normal Tyr levels. High blood Phe levels facilitate the blood-brain barrier exchange of Phe at the expense of other large neutral amino acids into the brain including Tyr and tryptophan. Therefore, high blood Phe results in high brain levels of Phe that may affect white matter (Dyer 1999) and reduced brain availability of the precursors Tyr and tryptophan for the neurotransmitter synthesis of dopamine and serotonin, respectively. Clinically, untreated PKU is characterized by severe intellectual disability, neurological problems, motor deficits, and behavioural problems (Blau et al. 2010; DeRoche and Welsh 2008; Jahja et al. 2016; Smith and Knowles 2000). Treatment, through a Phe-restricted diet plus amino acid supplements, tetrahydrobiopterin $\left(\mathrm{BH}_{4}\right.$, being a pharmacological chaperone protein of PAH) supplementation, or both, reduces blood Phe levels, which results in lower Phe levels and probably higher levels of neurotransmitter precursors such as Tyr and tryptophan (Blau et al. 2010; van Vliet et al. 2015, 2016). When started early after birth, treatment with dietary Phe restriction prevents intellectual disability, but, compared to healthy controls, PKU patients generally still have lower IQs and perform more poorly in several cognitive domains (Albrecht et al. 2009; Huijbregts et al. 2002a, c; Jahja et al. 2014; Moyle et al. 2007; Smith and Knowles 2000; Waisbren et al. 2007). The most frequently observed cognitive deficits in treated PKU are in executive functions (EFs), i.e. 'higher-order cognitive abilities that control and coordinate behaviour and constitute the driving force of goal-directed behaviour' (Christ et al. 2010; Huijbregts et al. 2002c, 2003; Moyle et al. 2007). Both cognitive impairment in treated PKU (AntenorDorsey et al. 2013; Blau et al. 2010; Huijbregts et al. 2002c) and internalizing mental health problems, such as anxiety, depression and mood swings (Anjema et al. 2011; Arnold et al. 1998; Cappelletti et al. 2013; Jahja et al. 2013;
Smith and Knowles 2000; Weglage et al. 2000) have repeatedly been associated with concurrent and historical blood Phe levels. However, questions remain regarding long-term consequences of elevated Phe in various periods of life on cognitive functioning in adults with PKU.

The key statements of the first European guidelines on PKU clearly show the lack of valuable data on the relation between outcome and metabolic control from adolescence onwards (van Spronsen et al. 2017). While there are some studies relating adult outcomes to history of metabolic control, and other studies indicating the existence of certain sensitive periods for elevated Phe with respect to cognitive development (Huijbregts et al. 2002b), none of these provide complete and conclusive evidence. Studies with repeated measurements are very scarce. One longitudinal study reported on neurocognitive functioning of 57 adult patients aged 19-41 years who were re-examined after a 5-year interval (Weglage et al. 2013). Patients had a lower IQ than controls, but psychomotor function and sustained attention were not different between PKU patients and controls at either time point. The authors further distinguished between participants younger and older than 32 years old: older patients had slower information processing than controls at both time points. Group differences did not change during the 5-year interval. High blood Phe levels in childhood and adolescence were related to poorer IQ, information processing and attention in adulthood. A limitation of this study of Weglage et al. (2013) was that both assessments took place during adulthood, so cognitive development could not be mapped from childhood onwards. A second longitudinal study assessed 14 PKU patients in childhood and early adulthood. Patients were 8-14 years at time point 1 and $22-28$ years at time point 2 . That study showed that differences in neuropsychological outcome between patients and controls became smaller throughout adolescence and adulthood, but did not disappear (Nardecchia et al. 2015). Regarding lifetime Phe, specifically those with Phe between 501 and $600 \mu \mathrm{mol} / \mathrm{L}(\mathrm{n}=3)$ performed worse than those with lifetime Phe below $500 \mu \mathrm{mol} / \mathrm{L}$ $(n=4)$. These authors also differentiated between patients with Phe below or above $600 \mu \mathrm{mol} / \mathrm{L}$ during the interval between the two time points: those with Phe above $600 \mu \mathrm{mol} / \mathrm{L}(\mathrm{n}=7)$ during the interval had poorer cognitive outcome as adults compared to controls $(n=14)$. This study provided evidence for an influence of adolescent or 'second decade of life' metabolic control on adult cognitive outcomes. A limitation of this study was that the authors could not control optimally for childhood Phe levels in their statistical analyses, as Phe levels were only included for the first 4 years of life, whereas critical stages of EFdevelopment occur later in childhood as well (Huijbregts et al. 2002b). So there is no strong evidence yet that adolescent or adult Phe levels truly influence adult outcome: it 
is still possible that childhood Phe levels, which are often related to Phe levels later in life, most strongly determine adult outcome.

The present study aimed to examine mental health, and development of executive functioning and executive motor control of PKU patients from childhood into adulthood in relation to historic metabolic control, distinguishing between different developmental stages and using different methodological approaches, compared to previous studies.

\section{Methods}

\section{Participants}

This study is part of the Dutch longitudinal multicentre PKU-COBESO study. Between February 1997 and October 1998, executive functioning and executive motor control were assessed in 67 children with PKU, aged 7-14 years (Huijbregts et al. 2002c). Patients that could be reached and who were willing to participate a second time were followed up between February 2012 and May 2015, at which point all of them were adults. At time point 1 (T1) and time point 2 (T2) identical tasks were performed, with additional tasks and questionnaires at T2 (Jahja et al. 2013). Patients were recruited through six Dutch university medical centres and on both occasions assessed in their treatment centre by the research staff. All patients were diagnosed by neonatal screening, which has been introduced in the Netherlands in September 1974, and treated early after birth. At both time points, T1 and T2, blood samples were taken on the day of neuropsychological testing in order to obtain concurrent Phe levels (conPhe). Historical Phe levels were collected from physicians' databases. Indices of dietary control (IDC) were calculated as the mean of all half-year median Phe levels until the day of testing. Thus, IDC1 was computed from birth until the day of testing at $\mathrm{T} 1$, and IDC2 (i.e. lifetime Phe) represented Phe levels from birth until the day of testing at $\mathrm{T} 2$. To create a difference score between both time points, i.e. to determine the increase in Phe between the two assessments, IDC1 was subtracted from IDC2 (i.e. IDC difference score). Mean of half-year median Phe levels between 0 and 12 years, between 13 and 17 , and $\geq 18$ years were also calculated.

Furthermore, based on the question whether different upper target Phe levels should be maintained during childhood (0-12 years) and thereafter, and the most frequently recommended upper target Phe level of $360 \mu \mathrm{mol} / \mathrm{L}$, patients were divided into groups: Phe $<360 \mu \mathrm{mol} / \mathrm{L}$ in childhood and Phe $<360 \mu \mathrm{mol} / \mathrm{L}$ from age 13 ('low-low' group); low Phe in childhood and high Phe $\geq 360 \mu \mathrm{mol} / \mathrm{L}$ from age 13 ('low-high' group); and high
Phe $\geq 360 \mu \mathrm{mol} / \mathrm{L}$ in childhood and high Phe from age 13 ('high-high' group).

\section{Measures}

The overall multicentre study (PKU-COBESO, Jahja et al. 2013) addressed cognitive, behavioural and social sequelae of early and continuously treated PKU patients in relation to history of metabolic control. A standardized testing protocol was used for each participant and took approximately $2.5 \mathrm{~h}$ to complete including breaks (Jahja et al. 2013). The following three computerized tasks of the Amsterdam Neuropsychological Tasks (ANT; De Sonneville 2014) were used to assess executive functioning and executive motor control on two different occasions, i.e. at T1 and T2.

In the Flanker (FL) interference task, which measures inhibitory control but more specifically interference suppression, stimuli consist of squares divided up into nine smaller squares. Participants must respond to the colour of the central smaller square, while ignoring the surrounding eight smaller squares (i.e. the flanker stimuli). When the central stimulus is blue, participants have to press the left mouse button; when the central stimulus is yellow, they have to press the right mouse button. In part 1 the flanker stimuli had a neutral colour (20 trials) or the same colour (20 trials, i.e. the compatible condition) as the central stimulus, while in part 2 the flankers had the same ( 40 trials, compatible condition) or the interfering (40 trials, incompatible condition) colour. The interfering colour meant to elicit a response with the other hand if it would have been the central stimulus colour (Huijbregts et al. 2002c). The differences in error percentage and reaction time (RT) between compatible and incompatible flanker stimuli in part 2 of the task were used to measure inhibitory control/ interference suppression.

The shifting attentional set-visual (SSV) task measures inhibitory control but more specifically inhibition of prepotent responding, and cognitive flexibility. On the screen a horizontal bar consisting of ten squares is presented. In task part 1 (40 trials, compatible condition), participants follow a green block which randomly moves across the bar to left or to right: movements to the left require left mouse button presses and movements to the right require right mouse button presses. In part 2 (40 trials, incompatible condition), the randomly moving block is red and a response is required opposite to the direction of the movement, i.e. movements to the left require right mouse button presses and vice versa. In part 3 (80 trials), the block changed randomly into green or red after each movement and participants switch between the rules of task parts 1 and 2 (i.e. compatible and incompatible responding) depending on the colour of the block after each random movement (Huijbregts et al. 2002a). This task requires inhibition of 
prepotent responding, when in task part 2 a switch has to be made from the automatic (compatible) response mode in task part 1 to the incompatible response mode, and it requires cognitive flexibility, when in task part 3, one has to switch between the two active response modes based on the cue provided by the stimulus. The differences in error percentages and RT between part 1 and 2 represented inhibition of prepotent responding and the differences between part 1 and 3 represented cognitive flexibility.

Executive motor control was measured with the pursuit (PU) task. Participants had to follow an asterisk, which randomly moved across the screen, by placing the mouse cursor as closely as possible on top of the asterisk for $2 \mathrm{~min}$, first with their non-dominant hand, then with their dominant hand (Huijbregts et al. 2003). Mean deviation from the moving target (i.e. accuracy of movement), and standard deviation of the trajectory that was followed (stability of movement) were used in analyses. A greater deviation or standard deviation indicated poorer executive motor control.

At T2, general/demographic information was collected and two questionnaires were administered. First, the Behavior Rating Inventory of Executive Function-Adult version (BRIEF-A) was used to measure executive functioning in daily life of adults (Roth et al. 2005). The questionnaire consists of 75 questions, assessing nine subdomains of executive functioning: Inhibit, Shift (Cognitive Flexibility), Emotional Control, Self-Regulation, Initiate, Working Memory, Plan/Organize, Organization of Materials, and Monitor. The subdomains Inhibit, Shift, Emotional Control and Self-Regulation together represent the Behavioral Regulation Index (BRI). Furthermore, the combined scores of the other five subdomains represent the MetaCognition Index (MCI). The Global Executive Composite (GEC) is the total score of all subdomains and represents the overall executive functioning in daily life. Continuous T-scores of the BRI, MI and GEC were used in statistical analyses. For descriptive purposes the following distinctions were used: participants have normal EFs when T-scores are below 50 . A T-score between 50 and 65 is considered increased or borderline, and a T-score above 65 indicates clinical significance (i.e. in the clinical range).

Second, the Adult Self-Report (ASR) of the Achenbach System of Empirically Based Assessment (Achenbach and Rescorla 2003) measured mental health problems. It is a norm-referenced questionnaire consisting of 102 items with 3-point rating scales suitable for adults. Six DSM-IV-oriented scales are provided: Depressive problems, Anxiety problems, Somatic problems, Avoidant Personality problems, Attention Deficit/Hyperactivity problems, and Antisocial Personality problems. Next to these subscales, the overall internalizing, externalizing and total problem score were also used. For the three overall scales, a score below
60 is considered normal, scores between 60 and 64 are in the borderline range, and scores above 64 are in the clinical range. Thus, higher scores represent more mental health problems.

\section{Statistical Analyses}

IBM SPSS Statistics 22nd version was used for statistical analyses. Associations between executive functioning and executive motor control at $\mathrm{T} 1$ and $\mathrm{T} 2$, and mental health at $\mathrm{T} 2$ on the one hand and several indicators of metabolic control on the other (i.e. conPhe at T2, IDC1, IDC2, IDC difference score, Phe $0-12,13-17$ and $\geq 18$ years) were investigated using one-tailed Pearson correlations and partial correlations to control for Phe $0-12$ years. Repeated measures analyses of variance were used to compare the 'low-high' groups at $\mathrm{T} 1$ and $\mathrm{T} 2$ on performance of the ANT-tasks measuring executive functioning and executive motor control. Z-scores of the EF measures at T1 and T2, calculated using age-appropriate performance scores from healthy controls in the PKU-COBESO study as norm reference, were used as dependent variables, where a low or negative Z-score indicated a better performance. Independent samples T-tests were conducted for comparisons of the 'low-high' groups on BRIEF and ASR scores at T2.

\section{Results}

\section{Metabolic Outcome}

Twenty-one patients (31\% of the original cohort; 6 male, 15 female) completed a neuropsychological assessment at two time points. The remaining $69 \%$ of the original cohort was lost to follow up. Patients were not under treatment anymore and/or were out of sight from physicians $(n=40)$ or were not willing to participate $(n=6)$, usually due to time constraints. IDC at T1 $(t(62)=1.1, p=0.29)$ and the pretreatment Phe $(t(64)=0.4, p=0.72)$ did not significantly differ between the 21 versus 46 patients, but conPhe at $\mathrm{T} 1$ was higher for the $69 \%$ that was lost $(t(49)=2.0, p=0.027)$. However, data regarding executive functioning was gathered for both groups at T1. The two groups did not differ on inhibitory control (FL and SSV), cognitive flexibility (SSV) and executive motor control (PU), indicating that the two groups had similar executive functioning at $\mathrm{T} 1$.

For those 21 patients who participated at $\mathrm{T} 2$, mean age at $\mathrm{T} 1$ was 10.4 years $(\mathrm{SD}=2.0)$ and 25.8 years $(\mathrm{SD}=2.3)$ at $\mathrm{T} 2$ (see Table 1 for descriptive statistics). According to the traditional classification for biochemical subtypes within the hyperphenylalaninemias (Blau et al. 2011), four patients were classified as having hyperphenylalaninemia (HPA, i.e. a pre-treatment Phe level 
Table 1 Descriptive statistics PKU, and 'low-high' groups

\begin{tabular}{lllll}
\hline & PKU $=21$ & Low-low $=3^{\text {a }}$ & Low-high $=11$ & High-high $=7$ \\
\hline Mean age T1 \pm SD (range) & $10.5 \pm 2.0(6.9-13.7)$ & $11.0 \pm 2.3(9.3-13.6)$ & $10.7 \pm 2.1(6.9-13.7)$ & $9.9 \pm 1.7(7.0-12.4)$ \\
Mean age T2 \pm SD (range) & $25.8 \pm 2.3(21.0-30.5)$ & $26.6 \pm 2.5(23.9-28.7)$ & $25.7 \pm 2.8(21.0-30.5)$ & $25.7 \pm 1.7(23.0-28.8)$ \\
Gender (male:female) & $6: 15$ & $0: 3$ & $5: 6$ & $1: 6$ \\
Socio-economic status: income & 7 Above average & Average & 5 Above average & 2 Above average \\
Socio-economic status: education & 14 Higher education & 3 Higher education & 7 Higher education & 4 Higher education \\
IQ & $102 \pm 13(71-120)$ & $103 \pm 14(88-115)$ & $103 \pm 9(92-120)$ & $101 \pm 18(71-120)$ \\
Diagnostic Phe measurement & $1300 \pm 925(120-3151)$ & $205 \pm 107(120-325)$ & $1252 \pm 805(450-3053)$ & $1844 \pm 904(750-3151)$ \\
Biochemical PKU phenotype & 4 HPA; 8 mild PKU; 9 & 3 HPA & 1 HPA; 6 mild PKU; 4 & 2 mild PKU; classical PKU \\
& classical PKU & & classical PKU & \\
BH4 responsive & 5 BH4 responsive & 2 BH4 responsive & 2 BH4 responsive & 1 BH4 responsive \\
Concurrent Phe T1 \pm SD (range) & $346 \pm 204(30-860)$ & $308(2$ missing) & $257 \pm 157(30-475)$ & $491 \pm 211(245-860)$ \\
IDC T1 \pm SD (range) & $315 \pm 92(192-548)$ & $326 \pm 53(294-388)$ & $252 \pm 42(192-327)$ & $409 \pm 84(320-548)$ \\
Concurrent Phe T2 \pm SD (range) & $719 \pm 351(259-1550)$ & $357 \pm 87(259-427)$ & $807 \pm 403(336-1550)$ & $735 \pm 241(345-1000)$ \\
IDC T2 \pm SD (range) & $464 \pm 138(276-743)$ & $291 \pm 15(276-306)$ & $424 \pm 79(322-547)$ & $602 \pm 112(446-743)$ \\
IDC difference score (IDC2 minus & $149 \pm 120(-82-330)$ & $-35 \pm-41(-82$ to -6$)$ & $172 \pm 93(52-322)$ & $194 \pm 112(-6-330)$ \\
IDC1) & & & &
\end{tabular}

BH4 tetrahydrobiopterin, IDC index of dietary control, $P K U$ phenotype based on diagnostic Phe measurement, $H P A$ hyperphenylalaninemia, Phe

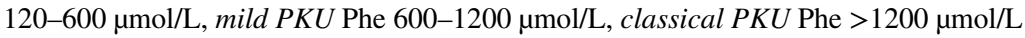

${ }^{a}$ The low-low group was excluded from statistical analyses because the sample size was too small

below $600 \mu \mathrm{mol} / \mathrm{L}$ ), eight as mild PKU (pre-treatment Phe between 600 and $1200 \mu \mathrm{mol} / \mathrm{L}$ ) and nine had classical PKU (pre-treatment Phe $\geq 1200 \mu \mathrm{mol} / \mathrm{L}$ ). Mean conPhe at $\mathrm{T} 1$ was $346 \mu \mathrm{mol} / \mathrm{L}(\mathrm{SD}=204)$ while at $\mathrm{T} 2$ this was $719 \mu \mathrm{mol} / \mathrm{L}(\mathrm{SD}=351)$. Mean IDC at $\mathrm{T} 1$ was $312 \mu \mathrm{mol} / \mathrm{L}(\mathrm{SD}=96)$ and IDC at T2 was $464 \mu \mathrm{mol} / \mathrm{L}$ $(\mathrm{SD}=138)$. Phe levels between 0 and 12 years, between
13 and 17 and $\geq 18$ years are reported in Table 1 . Correlations between Phe variables are displayed in Table 2. Phe concentrations significantly increased with age, as shown with repeated measures including Phe $0-12,13-17$ and $\geq 18$ years (Wilks' $\Lambda=0.35, F(2,19)=17.30, p<0.001$, $\eta^{2} p=0.65$ ). Within $\mathrm{T} 1$ and within $\mathrm{T} 2$, age did not significantly correlate with Phe. At $\mathrm{T} 1, \mathrm{BH}_{4}$ was not yet

Table 2 Pearson correlations between indicators of metabolic control

\begin{tabular}{|c|c|c|c|c|c|c|c|c|}
\hline & $\begin{array}{l}\text { Concur- } \\
\text { rent Phe } \\
\text { at } \mathrm{T} 1\end{array}$ & IDC at T1 & $\begin{array}{l}\text { Concurrent Phe } \\
\text { at } \mathrm{T} 2\end{array}$ & IDC at $\mathrm{T} 2$ & $\begin{array}{l}\text { IDC difference } \\
\text { score }\end{array}$ & Phe $0-12$ years & Phe $13-17$ years & Phe $\geq 18$ years \\
\hline $\begin{array}{l}\text { Concurrent Phe } \\
\text { at } \mathrm{T} 1\end{array}$ & 1.000 & & & & & & & \\
\hline IDC at T1 & $0.543 * *$ & 1.000 & & & & & & \\
\hline $\begin{array}{l}\text { Concurrent Phe } \\
\text { at } \mathrm{T} 2\end{array}$ & 0.221 & -0.117 & 1.000 & & & & & \\
\hline IDC at $\mathrm{T} 2$ & $0.529 * *$ & $0.520 * *$ & $0.570 * *$ & 1.000 & & & & \\
\hline $\begin{array}{l}\text { IDC difference } \\
\text { score }\end{array}$ & 0.170 & -0.174 & $0.747 * * *$ & $\mathbf{0 . 7 5 1} * * *$ & 1.000 & & & \\
\hline Phe $0-12$ years & $0.553 * *$ & $0.933 * * *$ & 0.041 & $0.693 * * *$ & 0.078 & 1.000 & & \\
\hline Phe $13-17$ years & $0.387^{+}$ & 0.234 & 0.488* & $0.877 * * *$ & $\mathbf{0 . 8 3 0}^{* * *}$ & $0.466 *$ & 1.000 & \\
\hline Phe $\geq 18$ years & $0.385^{+}$ & 0.170 & $0.778 * * *$ & $0.876 * * *$ & $0.879 * * *$ & $0.328+$ & $\mathbf{0 . 7 5 4} * * *$ & 1.000 \\
\hline
\end{tabular}

$p<0.05$ values are indicated in bold

$I D C$ index of dietary control, $T 1$ time point 1 in childhood, $T 2$ time point 2 in adulthood

${ }^{*} p<0.05 * * p<0.01 * * * p<0.001,{ }^{+} p<0.10$ (1-tailed) 
prescribed in the Netherlands. At T2, five patients used $\mathrm{BH}_{4}$ doses up to $20 \mathrm{mg} / \mathrm{kg}$ with a max of $1400 \mathrm{mg} /$ day.

Regarding the 'low-high' groups, three patients were allocated to the 'low-low' group (mean Phe $<360 \mu \mathrm{mol} / \mathrm{L}$ when $0-12$ years and from age 13 onwards), 11 patients were in the 'low-high' group (Phe $<360 \mu \mathrm{mol} / \mathrm{L}$ until age 12 and $\geq 360 \mu \mathrm{mol} / \mathrm{L}$ from age 13$)$ and seven patients were in the 'high-high' group (Phe $\geq 360 \mu \mathrm{mol} / \mathrm{L}$ in childhood and onwards). None of the patients had high values as a child and low values as an adult. Descriptive statistics and metabolic measurements for these groups are displayed in Table 1. Because the first group was too small, only the 'low-high' and 'high-high' groups were included in groupbased statistical analyses. The 'low-high' group consisted of one patient with hyperphenylalaninemia, six patients with mild PKU and four patients with classical PKU, while the 'high-high' group included two mild PKU patients and five patients with classical PKU. The pre-treatment Phe concentration $(t(16)=-1.5, p=0.17)$ and the PKU classification $\left(\chi^{2}=2.3, p=0.31\right)$ did not differ between the two groups. The socio-economic status, i.e. education and yearly income, of these two groups was similar, respectively $\chi^{2}=3.1, p=0.37$ and $\chi^{2}=1.7, p=0.89$.

\section{Participant Characteristics: Relationships, Education, Occupation, Income}

Sixteen out of 21 patients $(76 \%)$ had a long-term, romantic relationship, 13 have had two or more long-term, romantic relationships in the past. All patients completed high school. Eight patients (38\%) followed or completed higher vocational education and six patients (29\%) completed higher education (bachelor's or master's degree), which is comparable to the healthy Dutch population, according to the Dutch Central Bureau for Statistics (CBS 2016a). All patients had an occupation at T2, working 12-40 h/week. Seven patients $(33 \%)$ had a higher than average income and 12 out of 21 (57\%) were house owners of private property, similar to the Dutch population (CBS 2016b).

\section{Associations Between Metabolic Control, Executive Functioning and Mental Health}

Regarding inhibitory control/interference suppression (FLtask), partial correlations (controlling for Phe 0-12 years) showed that Phe 13-17 years, the IDC difference score and IDC2 were significantly associated with percentage errors at T2 (see Table 3 for associations between Phe and ANTtasks). The positive correlation between the IDC difference score and error percentage indicated that with a larger increase in Phe between childhood and adulthood, poorer performance was observed at $\mathrm{T} 2$.
Table 3 Partial correlations (Pearson, 1-tailed) between Phe and ANT

\begin{tabular}{|c|c|c|}
\hline & $r$ & $p$ \\
\hline \multicolumn{3}{|c|}{ Flanker-inhibitory control/interference suppression } \\
\hline \multicolumn{3}{|l|}{$\%$ errors $\mathrm{T} 2$} \\
\hline Phe $13-17$ years & 0.578 & 0.004 \\
\hline IDC difference & $\mathbf{0 . 5 3 3}$ & 0.008 \\
\hline IDC T2 & 0.436 & $\mathbf{0 . 0 2 7}$ \\
\hline \multicolumn{3}{|c|}{ Shifting attentional set-visual—cognitive flexibility } \\
\hline \multicolumn{3}{|l|}{$\%$ errors $\mathrm{T} 2$} \\
\hline IDC difference & 0.393 & 0.053 \\
\hline Phe $13-17$ years & 0.426 & 0.039 \\
\hline IDC T2 & 0.354 & 0.075 \\
\hline \multicolumn{3}{|l|}{ Reaction time $\mathrm{T} 2$} \\
\hline IDC T1 & 0.446 & 0.028 \\
\hline Phe $0-12$ years & 0.429 & $\mathbf{0 . 0 3 3}$ \\
\hline \multicolumn{3}{|c|}{ Pursuit-executive motor control } \\
\hline \multicolumn{3}{|c|}{ Mean deviation $\mathrm{T} 2$} \\
\hline IDC T1 & 0.490 & 0.012 \\
\hline Phe $0-12$ years & 0.404 & $\mathbf{0 . 0 3 5}$ \\
\hline \multicolumn{3}{|c|}{ Standard deviation $\mathrm{T} 2$} \\
\hline IDC T1 & 0.550 & 0.005 \\
\hline Phe $0-12$ years & 0.515 & 0.008 \\
\hline \multicolumn{3}{|c|}{$p<0.05$ values are indicated in bold } \\
\hline \multicolumn{3}{|c|}{ Note only significant correlations and (non-significant) trends shown } \\
\hline
\end{tabular}

Inhibition of prepotent responding (SSV-task) was not associated with any of the Phe indices. When measuring cognitive flexibility, the percentage of errors at $\mathrm{T} 2$ was significantly correlated with Phe 13-17 years while the correlation with the IDC difference score just failed to reach significance after controlling for Phe $0-12$ years. RT at T2 was associated with IDC1 and with Phe $0-12$ years.

Regarding executive motor control (PU-task), IDC1 and Phe $0-12$ years were significantly related to accuracy and stability of executive motor control at T2. These correlations indicated that high Phe in childhood were related to poorer executive motor control in adulthood.

The Behavioral Regulation Index (BRI) of the BRIEFA which was only measured at $\mathrm{T} 2$, was significantly associated with IDC1, Phe 0-12 years and IDC2 (see Table 4 for correlations between Phe and questionnaires). When controlling for Phe 0-12 years, the correlation with IDC2 became non-significant. The Global Executive Composite (GEC) was also related to Phe 0-12 years. These results demonstrate that childhood Phe is important for executive functioning in daily adult life.

With respect to mental health at T2, Depressive problems were related to Phe 0-12 years and to IDC2. Somatic problems had a significant relation with IDC1, Phe 
Table 4 Partial correlations (Pearson, 1-tailed) between Phe and behavior ratings

\begin{tabular}{|c|c|c|c|c|c|c|c|c|c|c|}
\hline & \multicolumn{2}{|c|}{ IDC T1 } & \multicolumn{2}{|l|}{ IDC T2 } & \multicolumn{2}{|c|}{ Phe $0-12$ years } & \multicolumn{2}{|c|}{ Phe $13-17$ years } & \multicolumn{2}{|c|}{ Phe $\geq 18$ years } \\
\hline & $r$ & $p$ & $r$ & $p$ & $r$ & $p$ & $r$ & $p$ & $r$ & $p$ \\
\hline \multicolumn{11}{|l|}{ BRIEF-A } \\
\hline Behavioural regulation index & 0.454 & 0.025 & 0.415 & 0.039 & 0.501 & 0.014 & 0.173 & 0.239 & 0.288 & 0.116 \\
\hline Global executive composite & 0.329 & 0.084 & 0.361 & 0.064 & 0.397 & 0.046 & 0.233 & 0.168 & 0.264 & 0.138 \\
\hline \multicolumn{11}{|l|}{ ASR } \\
\hline Depressive problems & 0.346 & 0.073 & 0.402 & 0.044 & 0.456 & 0.025 & 0.322 & 0.090 & 0.266 & 0.135 \\
\hline Anxiety problems & 0.255 & 0.146 & 0.213 & 0.191 & 0.289 & 0.115 & 0.151 & 0.268 & 0.076 & 0.378 \\
\hline Somatic problems & 0.609 & 0.003 & 0.451 & $\mathbf{0 . 0 2 6}$ & 0.721 & $\mathbf{0 . 0 0 0}$ & 0.247 & 0.153 & 0.226 & 0.177 \\
\hline Avoidant personality problems & 0.383 & 0.053 & -0.038 & 0.439 & 0.349 & 0.072 & -0.230 & 0.172 & -0.134 & 0.292 \\
\hline Attention deficit/hyperactivity problems & 0.567 & 0.006 & 0.351 & 0.070 & 0.572 & $\mathbf{0 . 0 0 5}$ & 0.169 & 0.244 & 0.211 & 0.193 \\
\hline Antisocial personality problems & 0.545 & 0.008 & 0.488 & 0.017 & 0.496 & 0.015 & 0.182 & 0.277 & 0.398 & 0.046 \\
\hline Internalizing problems & 0.512 & 0.012 & 0.130 & 0.298 & 0.568 & 0.006 & 0.007 & 0.488 & -0.096 & 0.347 \\
\hline Externalizing problems & 0.586 & 0.004 & 0.538 & 0.009 & 0.619 & 0.002 & 0.348 & 0.072 & 0.368 & 0.060 \\
\hline Total score & 0.524 & 0.011 & 0.342 & 0.074 & 0.593 & 0.004 & 0.174 & 0.238 & 0.162 & 0.254 \\
\hline
\end{tabular}

$p<0.05$ values are indicated in bold

$I D C$ index of dietary control, $T 1$ time point 1 in childhood, $T 2$ time point 2 in adulthood, BRIEF-A behavior rating inventory of executive function-adult version, $A S R$ adult self-report

0-12 years and IDC2. IDC1 and Phe 0-12 years were associated with Attention Deficit/Hyperactivity problems. Antisocial Personality problems were related to IDC1, Phe $0-12$ years, Phe $\geq 18$ years and IDC2. Overall internalizing problems were associated with IDC1 and Phe 0-12 years. The externalizing problem scale was related to IDC1, Phe 0-12 years and IDC2. However, when controlling for Phe 0-12 years, the correlations with Phe indices after childhood became non-significant. Finally, the overall total problem scale was again associated with IDC1 and Phe 0-12 years. The IDC difference score was not significantly related to the ASR scores.

\section{Multiple Testing}

Regarding the neuropsychological test outcomes, correction for multiple testing (seven IDCs times eight outcome measures) would result in non-significant correlations only, as a $p$ value of 0.0009 would be the criterion. We opted for calculating all these correlations as the different IDCs may provide (subtly) different information. Still, the IDCs were often strongly correlated (see Table 2). If one would retain only IDC at T1 and the IDC difference score, as they represent childhood Phe and Phe-change thereafter, and therefore seem the most meaningful indices, no significant correlations are retained either, as a $p$-value of 0.003 would be the criterion. Some could be retained by reducing the number of outcome measures, e.g. one per task. However, there is no evidence to support one should choose either RT, error rate, or stability of performance as main outcome measures of the tasks.
Questionnaire data showed stronger correlations with IDCs, with IDC1 and Phe0-12 correlations with the BRI of the BRIEF almost remaining significant after applying $p$-level corrections with a factor 4 , i.e. two indices of metabolic control (i.e. IDC1 and IDC difference score or Phe 0-12 and Phe >12), and two outcome measures (MCI and BRI indices). A number of results obtained on the ASR actually did remain significant after applying corrections with a factor 12 (i.e. two IDCs, six ASR-dimensions): somatic problems, attention deficit/hyperactivity problems, or 4 (i.e. two IDCs, two ASR-dimensions): internalizing and externalizing problems. The pattern of correlations indicated that especially those with IDC1 or Phe 0-12 were robust (Table 4). Further group comparisons were made (see below) in order to link results to upper target Phe levels (for different age periods) according to current treatment guidelines, and to gain more insight into development of cognition and mental health in PKU patients relative to healthy controls.

\section{Comparison 'Low-High' and 'High-High' Groups}

For inhibitory control/interference suppression (FL-task), repeated measures analyses of variance did not show significant effects for time or group. Also, there was no interaction effect. On the SSV-task, there were no significant effects for inhibition of prepotent responding. Concerning cognitive flexibility, the 'low-high' group was significantly faster than the 'high-high' group at both time points $\left(F(1,15)=8.0, p=0.013, n_{p}^{2}=0.349\right)$ (see Fig. 1). Although descriptive statistics showed better Z-scores on 


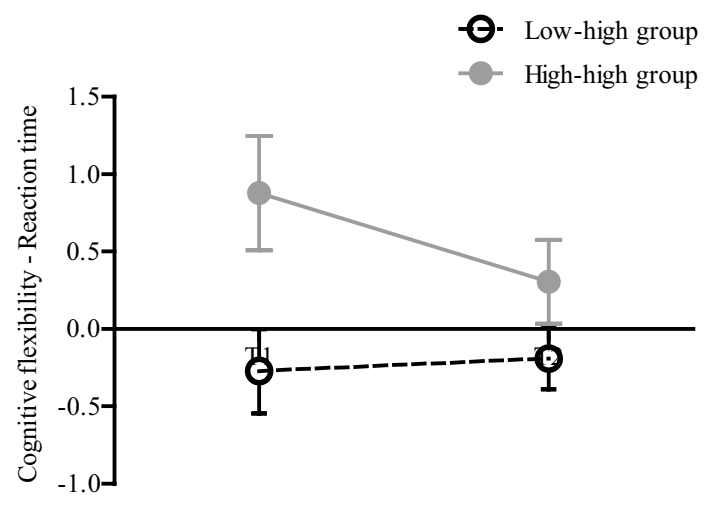

Fig. 1 Reaction time of cognitive flexibility. Group effect was significant: at both time points the low-high group performed faster than the high-high group

cognitive flexibility for all patients at $\mathrm{T} 2$ compared to the age-appropriate norm, time and interaction effects were not significant. For executive motor control (PU-task) there was a significant effect for time. All participants improved over time compared to the age-appropriate norm: they had a more accurate $\left(F(1,16)=5.5, p=0.033, n^{2}=0.255\right)$ and stable $\left(F(1,16)=4.5, \quad p=0.050, \quad n_{p}^{2}=0.219\right)$ executive motor control at $\mathrm{T} 2$. Also the group effect was significant for stability of movement: the 'low-high' group had a more stable executive motor control than the 'high-high' group $\left(F(1,16)=5.6, p=0.031, n^{2}=0.259\right)$ (see Fig. 2).

Regarding the BRIEF scales on executive functioning in daily life, there were no statistically significant differences between the 'low-high' $(\mathrm{n}=11)$ and 'high-high' $(\mathrm{n}=7)$ groups. Finally, with respect to mental health as measured by the ASR scales, the 'high-high' group reported significantly more Somatic problems than the 'low-high' group $(t(14)=-3.8, p=0.002)$. When examining the descriptive statistics, two patients from the 'low-high' group (18\%), scored in the borderline range of the internalizing scale and had a lifetime Phe of 420 and $514 \mu \mathrm{mol} / \mathrm{L}$ respectively. Two patients (29\%) from the 'high-high' group were in the clinical range with lifetime Phe of 446 and $678 \mu \mathrm{mol} / \mathrm{L}$. The latter patient with the highest Phe also scored in the clinical range of the externalizing and overall total problem scale. This patient also scored in the clinical range of the BRIEF. The patients in the normal range had similar lifetime and childhood Phe as those in the borderline and clinical range.

\section{Discussion}

The present study provided evidence for long-term effects of elevated childhood Phe on cognitive functioning and mental health during adulthood, although specifically for a number of cognitive indices more recent Phe levels, particularly Phe level between 13 and 17 years of age, were also associated with outcome, even after controlling for childhood Phe. Results confirmed that Phe levels increase with age. Phe levels increased in adolescence and were the highest in adulthood. This is consistent with results of previous studies (e.g. Walter et al. 2002; Walter and White 2004) and may be due to the fact that recommended treatment targets are higher in adolescence and adulthood compared to childhood (Blau et al. 2010; Weglage et al. 2013). A larger increase from childhood to adult Phe levels was associated with poorer inhibitory control/interference suppression and cognitive flexibility, as measured with the ANT, during adulthood.

Examining the changes in outcome between childhood and adulthood showed that executive motor function improved over time for PKU patients relative to the norm reference of healthy controls, whereas this was not observed for inhibitory control and cognitive flexibility. These last results are in line with findings from the study of Weglage et al. (2013), who concluded that in 5 years' time cognitive performance of adult patients remained stable, despite an increase in Phe levels. The present study showed that such stability of relative deficits apparently extends
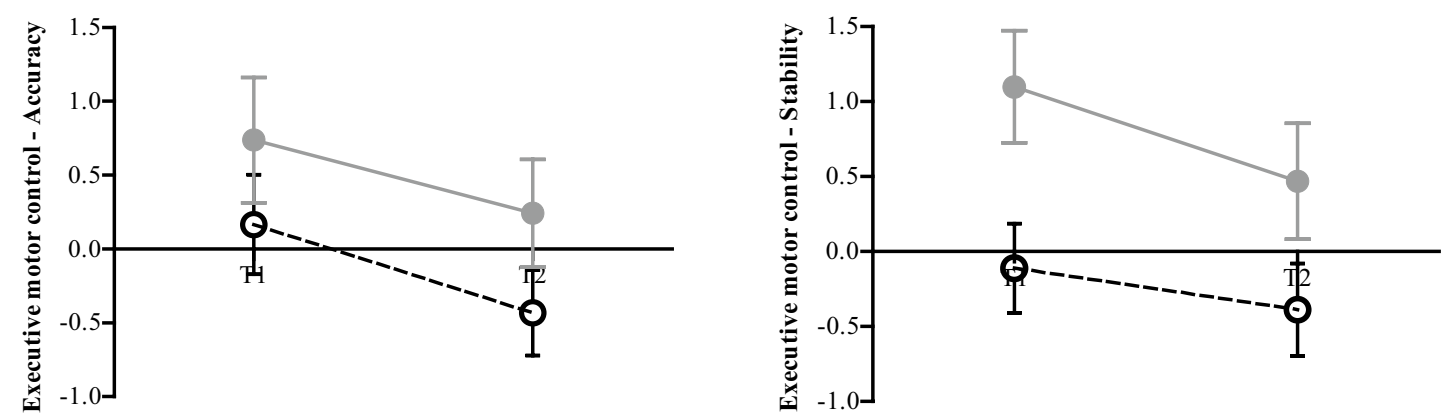

Low-high group High-high group

Fig. 2 Executive motor control: accuracy and stability of movement. Time effect was significant for accuracy and stability: all participants improved over time. Group effect was significant for stability only: the low-high group had a more stable executive motor control than the high-high group 
over a period of 10-15 years. In line with findings from Nardecchia et al. (2015) we showed a significant improvement relative to healthy controls in executive motor control between childhood/adolescence and adulthood. Nardecchia et al. (2015) reported relative improvements for other cognitive domains as well, incorporating a period between test occasions that was almost as long as ours, but in a sample that was smaller than ours. Differences may be related to measurement tools and sample characteristics. Our sample, for instance, consisted predominantly of patients at the higher end of the socio-economic status spectrum. Moreover, the 21 patients with $\mathrm{T} 2$ assessments did not differ significantly from healthy controls at T1 on the FI- and SSVtasks, which is in contrast with findings reported for the entire PKU sample at T1 (Huijbregts et al. 2002a, c). Even though the 21 patients did not differ from the 46 patients lost to follow-up regarding EF at $\mathrm{T} 1$ either, the evidence indicating that the T2 PKU group is relatively "well-off" or relatively mildly affected is more convincing than the evidence showing a lack of differences between patients that did versus those that did not participate at $\mathrm{T} 2$. This should be considered when interpreting our study results.

An explanation for the stability or improvement of cognition among PKU patients in both studies might lie in good or good enough treatment compliance and subsequent low Phe levels after childhood. The fact that the mean Index of Dietary Control at T2 (i.e. lifetime Phe level) was $464 \mu \mathrm{mol} / \mathrm{L}$, with a range of $276-743 \mu \mathrm{mol} / \mathrm{L}$ seems to support this notion. Despite the fact that Phe levels increased after childhood, these may still have been sufficiently low to allow patients to 'close' the gap with their healthy counterparts or at least not worsen compared to them. Considering this indicator of metabolic control, further patient characteristics, e.g. on education, employment, and romantic relationships, and the fact that this selection of patients did not differ from controls at $\mathrm{T} 1$, it cannot be ruled out either that our findings, and those of Nardecchia et al. (2015) and Weglage et al. (2013), are positively biased and that this bias extends to domains beyond metabolic control.

Relatively good cognitive and psychosocial development of individuals with PKU over longer periods of time may depend upon their childhood Phe levels. Unfortunately, this could not be comprehensively studied by Nardecchia et al. (2015) who only used Phe from the first 4 years of life as covariate for the negative association between lifetime Phe and executive functioning whilst critical periods for EF-development are known to occur beyond the age of four (Huijbregts et al. 2002b). This is particularly unfortunate as we found evidence for the importance of childhood Phe levels (i.e. between 0 and 12 years) with two different statistical approaches. First, we investigated whether Phe levels from different developmental stages were differentially associated with our neurocognitive and mental health outcome measures. Results showed that generally childhood Phe as measured by IDC at T1 or Phe between 0 and 12 years was solely or more strongly associated with the outcome measures at T2 (i.e. in adulthood). With two exceptions regarding higher-order executive functioning, which is known to continue development in the second decade of life, associations with post-childhood Phe levels were non-significant after controlling for childhood Phe. Second, we created groups of PKU patients based on Phe levels during childhood and thereafter, and compared outcomes during adulthood. As a cut-off for group assignment we took a Phe level of $360 \mu \mathrm{mol} / \mathrm{L}$, which is the recommended upper target Phe level worldwide for children up to the age of 12 (van Spronsen et al. 2017; Vockley et al. 2014), and, in the USA, also for the period thereafter (Vockley et al. 2014). As there were no patients going from high levels during childhood to low levels during adolescence and beyond, and only three patients in the 'low-low' group, we compared two groups: the 'low-high' group (Phe $<360 \mu \mathrm{mol} / \mathrm{L}$ until age 12 and $\geq 360 \mu \mathrm{mol} / \mathrm{L}$ from age 13) and the 'high-high' group (Phe $\geq 360 \mu \mathrm{mol} / \mathrm{L}$ throughout life). The 'low-high' group performed better on a number of tasks than the 'high-high' group, specifically with respect to cognitive flexibility and executive motor control, and also had better mental health outcomes as adults. These findings suggest that those patients who had low Phe in childhood had better outcome in adulthood than those who already had high Phe in their first 12 years of life.

In our study, adult mental health problems were also exclusively or most strongly related to elevated Phe in childhood. Phe between 0 and 12 years was related to Depressive, Somatic, Attention Deficit/Hyperactivity, Antisocial Personality problems, and the overall internalizing, externalizing and total problem scale. Associations between mental health problems and later Phe indices were no longer significant after controlling for childhood Phe. Brumm et al. (2010) demonstrated a relationship between severity of behavioural symptoms and timing and degree of exposure to Phe. They concluded that children with high Phe in earlier years were more likely to be affected and had more severe mental health problems. Our results are consistent with their finding.

It may be considered a limitation of this study that many statistical tests have been carried out. After correction for multiple testing by using adjusted $p$ values many significant results were lost. However, a pattern of results remained evident indicating a larger influence of childhood Phe levels (Phe 0-12 years, IDC1, Phe low-high vs. Phe high-high) compared to Phe levels later in life. Also, there are a number of arguments supporting the use of multiple statistical tests. Generally, two broad sets of analyses have been used: correlations between indices of metabolic control and outcome measures, and group-based analyses involving 
comparisons between PKU patients with Phe levels below the recommended upper target limit as a child ( $0-12$ years, below $360 \mu \mathrm{mol} / \mathrm{L}$ ) and higher levels thereafter and PKU patients with Phe levels higher than $360 \mu \mathrm{mol} / \mathrm{L}$ throughout life. Although the correlations were corrected for childhood Phe levels, which already made results indicative of the relative influence of Phe during different stages of life, the added value of the group-based analyses is that they relate possible differential influences of Phe during different stages of life to treatment guidelines (i.e. the upper target Phe level of $360 \mu \mathrm{mol} / \mathrm{L}$ ). The different IDCs that were included in the correlational analyses were often relatively strongly related, but it was still considered important to investigate not only IDC until T1 and until T2 but also to distinguish Phe levels between 0 and 12, 13 and 17 years and for 18 years and older because these periods are distinguished in treatment guidelines as well. Furthermore, results showed a potential influence of change in Phe levels between childhood and thereafter that could not be captured by analyses using absolute Phe levels. Thus, it may be concluded that results of different tests were complementary. Another, related limitation of the present study is that strong evidence for further specificity in the strengths and weaknesses profile of PKU patients cannot be provided. For this, we would have to include even more tasks (assessing RT and error rate) and (informant-rated) questionnaires, preferably in a larger sample. In general, however, clear and converging evidence for the continued influence of childhood Phe on adult cognitive (EF-) and behavioural outcomes was provided.

The most important limitation of our study is probably that our sample seemed positively biased and therefore not entirely representative of the "average" adult PKU patient. Because we missed many patients who had participated as a child, we also had a small sample size. This would render the outcome of further comparisons unreliable, e.g. between $\mathrm{BH}_{4}$ users and non-users, or a group-based analysis including the 'low-low' group. Whereas considering the rarity of PKU the sample size is still acceptable and comparable to other studies using a follow-up design in PKU, future studies should seek for ways or strategies to enhance more comprehensive inclusion of patients. Another concern may be the inclusion of different subtypes of PKU, making this sample heterogeneous. However, this enabled us to examine the effect of lower Phe and this was taken into account when analysing the 'low-high' and 'high-high' groups: there was no difference in type of classification between the two groups. A different type of limitation lies in the choice of Phe levels to represent metabolic control in different stages of development. As noted, we used 0-12, $13-17, \geq 18$ years, to differentiate between different developmental stages. However, the exact age ranges representing different developmental and possibly critical stages for cognitive and psychosocial development are unknown. Whereas we made informed choices and examined Phe levels during different time windows as well (e.g. the average Phe level between IDC at T1 and IDC at T2, which had a correlation of approximately $r=0.9$ with Phe 13-17 years and similar correlations as Phe 13-17 years with outcome measures), more general knowledge on developmental milestones or critical developmental stages for EF and psychosocial functioning would be very helpful in this respect. A final limitation lies in the possible underestimation of their own mental health problems by PKU patients. Although it is relatively custom to use self-reports in order to assess mental health, a more comprehensive assessment (using other informants and/or expert interviews) would be advisable for future studies.

In summary, our study showed that executive functioning in PKU patients is mostly stable over time from childhood into young adulthood, with some improvements relative to controls observed as well. Furthermore, long-term effects of elevated childhood Phe on cognitive and mental health outcome later in life were observed, supporting the notion that childhood Phe should be below the recommended treatment target of $360 \mu \mathrm{mol} / \mathrm{L}$ for better outcome in adulthood. Although the influence of childhood Phe levels was generally the strongest, the extent of the increase in Phe levels after childhood and Phe levels between 13 and 17 years of age were also related to some aspects of adult EF. With regard to this, we cannot entirely rule out the influence of later Phe levels as participants were almost exclusively characterized by good metabolic control from birth onwards and had grown up in social and socio-economic environments that may be considered protective or beneficial for cognitive and psychosocial development.

Acknowledgements The authors thank all PKU patients and controls for participating, and the psychology and MD students for assisting in this study. In addition, we thank all physicians for their full cooperation, the nursing staff, and the metabolic departments of all collaborating hospitals.

Funding This work was supported by the Dutch PKU Research Foundation, NutsOhra Fund, and the Division of Metabolic Diseases of the Beatrix Children's Hospital of the University Medical Center Groningen, The Netherlands. The sponsors were not involved in the study design, data collection, analysis and interpretation of data, writing of the report, and the decision to submit the manuscript for publication.

\section{Compliance with ethical standards}

Conflict of interest R. Jahja has received honoraria as a speaker and consultant for Merck Serono. F. J. van Spronsen was a member of various Scientific Advisory Boards of Merck Serono, is a member of Scientific Advisory Boards of Danone, APR and Biomarin and has received grants for research purpose of both Merck Serono (later Biomarin) and Nutricia. Further he has received consultation fees and speaker relation fees from Merck Serono, Biomarin, Nutricia and Vi- 
taflo. He has assisted in the design of clinical studies using products manufactured by Biomarin. He chairs the Scientific Advisory Board of the ESPKU and is the chairman of the group developing guidelines on PKU in Europe. L. M. J. de Sonneville declares receipt of consultation fees as consultant of Danone, FrieslandCampina, and Global Pharma Consultancy. A. M. Bosch is a member of Scientific Advisory Boards of Merck Serono and Danone, and was also involved in developing guidelines on PKU in Europe. S. C. J. Huijbregts has participated in strategic advisory boards and received honoraria as a consultant and/ or speaker for Merck Serono SA, Biomarin, and Nutricia. He is also a member of the group developing guidelines on PKU in Europe. J. J. van der Meere, C. E. M. Hollak, M. E. Rubio-Gozalbo, M. C. G. J. Brouwers, F. C. Hofstede, M. C. de Vries, M. C. H. Janssen, A. T. van der Ploeg, and J. G. Langendonk declare that they have no conflict of interest.

Ethical approval All procedures performed were in accordance with the ethical standards of the institutional and national research committee and with the 1964 Helsinki Declaration and its later amendments or comparable ethical standards.

Informed consent Informed consent was obtained from all participants for being included in the study.

Open Access This article is distributed under the terms of the Creative Commons Attribution 4.0 International License (http:// creativecommons.org/licenses/by/4.0/), which permits unrestricted use, distribution, and reproduction in any medium, provided you give appropriate credit to the original author(s) and the source, provide a link to the Creative Commons license, and indicate if changes were made.

\section{References}

Achenbach TM, Rescorla LA (2003) Manual for the ASEBA adult forms \& profiles. University of Vermont, Research Center for Children, Youth and Families, Burlington

Albrecht J, Garbade SF, Burgard P (2009) Neuropsychological speed tests and blood phenylalanine levels in patients with phenylketonuria: a meta-analysis. Neurosci Biobehav Rev 33:414-421

Anjema K, van Rijn M, Verkerk PH, Burgerhof JG, Heiner-Fokkema MR, van Spronsen FJ (2011) PKU: high plasma phenylalanine concentrations are associated with increased prevalence of mood swings. Mol Genet Metab 104:231-234

Antenor-Dorsey JA, Hershey T, Rutlin J, Shimony JS, McKinstry RC, Grange DK, Christ SE, White DA (2013) White matter integrity and executive abilities in individuals with phenylketonuria. Mol Genet Metab 109:125-131

Arnold GL, Kramer BM, Kirby RS, Plumeau PB, Blakely EM, Sanger Cregan LS, Davidson PW (1998) Factors affecting cognitive, motor, behavioral and executive functioning in children with phenylketonuria. Acta Paediatr 87:565-570

Blau N, van Spronsen FJ, Levy HL (2010) Phenylketonuria. Lancet 376:1417-1427

Blau N, Hennermann JB, Langenbeck U, Lichter-Konecki U (2011) Diagnosis, classification, and genetics of phenylketonuria and tetrahydrobiopterin (BH4) deficiencies. Mol Genet Metab 104(Suppl):S2-S9

Brumm VL, Bilder D, Waisbren SE (2010) Psychiatric symptoms and disorders in phenylketonuria. Mol Genet Metab 99(Suppl 1):S59-S63
Cappelletti S, Cotugno G, Goffredo BM, Nicolo R, Bernabei SM, Caviglia S, Di Ciommo V (2013) Cognitive findings and behavior in children and adolescents with phenylketonuria. J Dev Behav Pediatr 34:392-398

CBS (2016a) StatLine. https://www.cbs.nl/nl-nl/cijfers\#filter=all (2016)

CBS (2016b) StatLine: Arbeidsdeelname; regionale indeling 2015. CBS, Den Haag/Heerlen. http://statline.cbs.nl/Statweb/publication/? VW $=\mathrm{T} \& \mathrm{DM}=$ SLNL $\& \mathrm{PA}=83360 \mathrm{NED} \&$ $\mathrm{D} 1=0,15 \& \mathrm{D} 2=0,13 \& \mathrm{D} 3=0,101-493 \& \mathrm{D} 4=1 \& \mathrm{HD}=160518$ $1003 \& \mathrm{HDR}=\mathrm{G} 3, \mathrm{G} 1, \mathrm{~T} \& \mathrm{STB}=\mathrm{G} 2$ (2016)

Christ SE, Huijbregts SC, de Sonneville LM, White DA (2010) Executive function in early-treated phenylketonuria: profile and underlying mechanisms. Mol Genet Metab 99(Suppl 1):S22-S32

De Sonneville L (2014) Handboek ANT. Amsterdamse Neuropsychologische Taken. Boom Test Uitgevers, Amsterdam

DeRoche K, Welsh M (2008) Twenty-five years of research on neurocognitive outcomes in early-treated phenylketonuria: intelligence and executive function. Dev Neuropsychol 33:474-504

Dyer CA (1999) Pathophysiology of phenylketonuria. Mental Retard Dev Disabil Res Rev 5:104-112

Huijbregts S, de Sonneville L, Licht R, Sergeant J, van Spronsen F (2002a) Inhibition of prepotent responding and attentional flexibility in treated phenylketonuria. Dev Neuropsychol 22:481-499

Huijbregts SC, de Sonneville LM, Licht R, van Spronsen FJ, Verkerk PH, Sergeant JA (2002b) Sustained attention and inhibition of cognitive interference in treated phenylketonuria: associations with concurrent and lifetime phenylalanine concentrations. Neuropsychologia 40:7-15

Huijbregts SC, de Sonneville LM, van Spronsen FJ, Licht R, Sergeant JA (2002c) The neuropsychological profile of early and continuously treated phenylketonuria: orienting, vigilance, and maintenance versus manipulation-functions of working memory. Neurosci Biobehav Rev 26:697-712

Huijbregts SC, De Sonneville LM, Van Spronsen FJ, Berends IE, Licht R, Verkerk PH, Sergeant JA (2003) Motor function under lower and higher controlled processing demands in early and continuously treated phenylketonuria. Neuropsychology 17:369-379

Jahja R, Huijbregts SC, de Sonneville LM, van der Meere JJ, Bosch AM, Hollak CE, Rubio-Gozalbo ME, Brouwers MC, Hofstede FC, de Vries MC, Janssen MC, van der Ploeg AT, Langendonk JG, van Spronsen FJ (2013) Mental health and social functioning in early treated phenylketonuria: the PKU-COBESO study. Mol Genet Metab 110(Suppl):S57-S61

Jahja R, Huijbregts SC, de Sonneville LM, van der Meere JJ, van Spronsen FJ (2014) Neurocognitive evidence for revision of treatment targets and guidelines for phenylketonuria. J Pediatr 164(895-899):e2

Jahja R, van Spronsen FJ, de Sonneville LM, van der Meere JJ, Bosch AM, Hollak CE, Rubio-Gozalbo ME, Brouwers MC, Hofstede FC, de Vries MC, Janssen MC, van der Ploeg AT, Langendonk JG, Huijbregts SC (2016) Social-cognitive functioning and social skills in patients with early treated phenylketonuria: a PKUCOBESO study. J Inherit Metab Dis 39:355-62

Moyle JJ, Fox AM, Arthur M, Bynevelt M, Burnett JR (2007) Metaanalysis of neuropsychological symptoms of adolescents and adults with PKU. Neuropsychol Rev 17:91-101

Nardecchia F, Manti F, Chiarotti F, Carducci C, Carducci C, Leuzzi V (2015) Neurocognitive and neuroimaging outcome of early treated young adult PKU patients: A longitudinal study. Mol Genet Metab 115:84-90

Roth RM, Isquith PK, Gioia GA (2005) BRIEF-A. Behavior rating inventory of executive function-adult version. Psychological Assessment Resources, Inc, Lutz 
Smith I, Knowles J (2000) Behaviour in early treated phenylketonuria: a systematic review. Eur J Pediatr 159(Suppl 2):S89-S93

van Vliet D, Bruinenberg VM, Mazzola PN, van Faassen MH, de Blaauw P, Kema IP, Heiner-Fokkema MR, van Anholt RD, van der Zee EA, van Spronsen FJ (2015) Large neutral amino acid supplementation exerts its effect through three synergistic mechanisms: proof of principle in phenylketonuria mice. PLoS ONE 10:e 0143833

van Vliet D, Bruinenberg VM, Mazzola PN, van Faassen MH, de Blaauw P, Pascucci T, Puglisi-Allegra S, Kema IP, Heiner-Fokkema MR, van der Zee EA, van Spronsen FJ (2016) Therapeutic brain modulation with targeted large neutral amino acid supplements in the Pah-enu2 phenylketonuria mouse model. Am J Clin Nutr 104:1292-1300

van Spronsen F, van Wegberg A, Ahring K, Bélanger-Quintana A, Blau N, Bosch A, Burlina A, Campistol J, Feillet F, Giżewska M, Huijbregts S, Kearney S, Leuzzi V, Maillot F, Muntau A, Trefz F, van Rijn M, Walter J, MacDonald A (2017) Key European guidelines for the diagnosis and management of patients with phenylketonuria. Lancet Diabetes Endocrinol. doi:10.1016/ S2213-8587(16)30320-5

Vockley J, Andersson HC, Antshel KM, Braverman NE, Burton BK, Frazier DM, Mitchell J, Smith WE, Thompson BH, Berry SA,
American College of Medical Genetics and Genomics Therapeutics Committee (2014) Phenylalanine hydroxylase deficiency: diagnosis and management guideline. Genet Med 16:188-200

Waisbren SE, Noel K, Fahrbach K, Cella C, Frame D, Dorenbaum A, Levy H (2007) Phenylalanine blood levels and clinical outcomes in phenylketonuria: a systematic literature review and meta-analysis. Mol Genet Metab 92:63-70

Walter JH, White FJ (2004) Blood phenylalanine control in adolescents with phenylketonuria. Int J Adolesc Med Health 16:41-45

Walter JH, White FJ, Hall SK, MacDonald A, Rylance G, Boneh A, Francis DE, Shortland GJ, Schmidt M, Vail A (2002) How practical are recommendations for dietary control in phenylketonuria? Lancet 360:55-57

Weglage J, Grenzebach M, Pietsch M, Feldmann R, Linnenbank R, Denecke J, Koch HG (2000) Behavioural and emotional problems in early-treated adolescents with phenylketonuria in comparison with diabetic patients and healthy controls. J Inherit Metab Dis 23:487-496

Weglage J, Fromm J, van Teeffelen-Heithoff A, Moller HE, Koletzko B, Marquardt T, Rutsch F, Feldmann R (2013) Neurocognitive functioning in adults with phenylketonuria: results of a long term study. Mol Genet Metab 110(Suppl):S44-S48 